\title{
Combined endoscopic endonasal and transcranial approach to a recurrent frontal sinus mucocele
}

\begin{abstract}
The frontal mucocele remains a disease difficult to treat even in the era of functional endoscopic sinus surgery (FESS). Indeed, recurrence it is a typical complication of both endoscopic endonasal and transcranial approach or as a consequence of facial fracture. The modern endoscopic endonasal approach to the frontal sinus allows the marsupialization of the pathology in all those cases in which the mucocele is reached via the route of nasal drainage and is optimal when the location of the mucocele is medial. After infection or as a result of their invasion and growth, mucoceles of the frontal sinus can give rise to intracranial and orbital complications. The most versatile and used transcranial approach is considered the Sinus Fat Obliteration (SFO). We describe the case of a 47years old patient who under went FESS and OFSO for bilateral recurrence of mucocele within a megafrontal-sinus. The lesion reached the left supra orbital region with erosion of the roof of the ipsilateral orbit. The endoscopic approach, in our experience, it is necessary in the forms of mucocele complicated by orbital medial and superior extension. Instead, in the presence of a mega-frontal-sinus, of a lateral extension of the mucocele as well and the clinical history of relapses of the disease is also indicated the transcranial surgical approach. The Sinus Fat Obliteration (SFO) minimizes the risk of complications with no need for a postoperative neurointensive unit stay compared to the intervention of cranialization. In fact the patient object of this work had a regular postoperative course and was discharged on the sixth day with excellent aesthetic results.
\end{abstract}

Keywords: frontalsinusitis, mucocele, surgery, osteotomy, fatobliteration, surgical flaps, otorhinolaryngologic surgical procedures/methods
Volume 2 Issue I - 2015

\author{
Domenico Di Maria,' Matteo De Notaris, ${ }^{2}$ \\ Luigi Malafronte,' Angelo Maria Lannarone,' \\ Eugenio D avenia,' Giuseppe Catapano ${ }^{2}$ \\ 'ENT Department, "G. Rummo" Hospital, Italy \\ ${ }^{2}$ NeurosurgeryDepartment "G. Rummo" Hospital, Italy
}

Correspondence: Domenico Di Maria, ISAMED Center,Via Francesco Compagna 21, Campania, Italy, Tel +39082450632, Email domenicodimaria@gmail.com

Received: January 02, 20I5 | Published: January 13, 2015
Abbreviations: FESS, Functional Endoscopic Sinus Surgery; SFO, Sinus Fat Obliteration

\section{Introduction}

i. The sinus mucocele is an accumulation of sterile secretions inside a closed cavity (paranasal sinuses). The stagnation of secretions in the sinuses may promote bacterial growth and determine later a pyocele.

ii. The frontal mucocele remains a disease difficult to treat even in the era of functional endoscopic sinus surgery (FESS). Indeed, recurrence it is a typical complication of both endoscopic endonasal and transcranial approach or as a consequence of facial fracture.

iii. The frontal sinus external obliteration has been the method of choice as an evolution of the Lynch's and Lathrop's technique that were burdened with a relatively high recurrence rate.

iv. The modern endoscopic endonasal approach to the frontal sinus allows the marsupialization of the pathology in all those cases in which the mucocele is reached via the route of nasal drainage and is optimal when the location of the mucocele is medial.

v. After infection or as a result of their invasion and growth, mucoceles of the frontal sinus can give rise to intracranial and orbital complications..$^{1,2}$

vi. More recently, using the Draf technique is possible to reach also the lateral extensions, but, in specific cases, when there are orbital invasion (erosion of the floor or medial wall of the orbit), when there is a mega frontal sinus or when the endoscopic endonasal approach was followed by recurrence, a combined endoscopic endonasal and transcranial approach can be considered the first choice. ${ }^{3-7}$

vii. The most versatile and used transcranial approach is considered the Sinus Fat Obliteration (SFO).

\section{Case presentation}

a. We describe the case of a 47 years old patient who about 12 years before undergoing FESS for massive nasal polyposis (type IV according Stammberger) and then about 6 years before to a second approach by Lynch technique for a right frontal mucocele. The patient, about a year ago, was evaluated by ENT surgeon since complained frontal headache and swelling of the left eyebrow.

b. Then he underwent FESS with marsupialization of the mucocele by FESS.

c. No postoperative complications were observed.

d. Eight months after the symptoms recurred so the patient underwent diagnostic nasal endoscopy that showed a large area of fibrosis at the region of the left frontal-nasal duct. A CT scan showed bilateral recurrence of mucocele within a mega-frontalsinus. The lesion reached the left supraorbital region with erosion of the roof of the ipsilateral orbit (Figure 1).

e. After some days the patient developed a ptosis of the left eye.

f. For this reason, he was submitted to a brain MRI for the evaluation of the extension of the mucocele and of the involvement of the frontal sinus posterior wall and the dura mater (Figures $2 \& 3$ ). 
g. Concerning the surgery, a combined endonasal and transcranial approach was selected as the best strategy for the patient.

h. The first time was FESS. We proceeded to interruption of the synechiae at level of nasal-frontal duct to reach the region of the mucus-piocele that overlooked the left orbit (Figure 4).

i. So it was facilitated the drainage of mucus-pus nasally by the help of the second operator who performed repeated maneuvers of eye's pressure (Figure 5)

j. The purulent material was collected and sent for bacteriological and culture examination for a targeted postoperative antibiotic therapy.

k. After that a standard SFO was performed. ${ }^{4-6}$

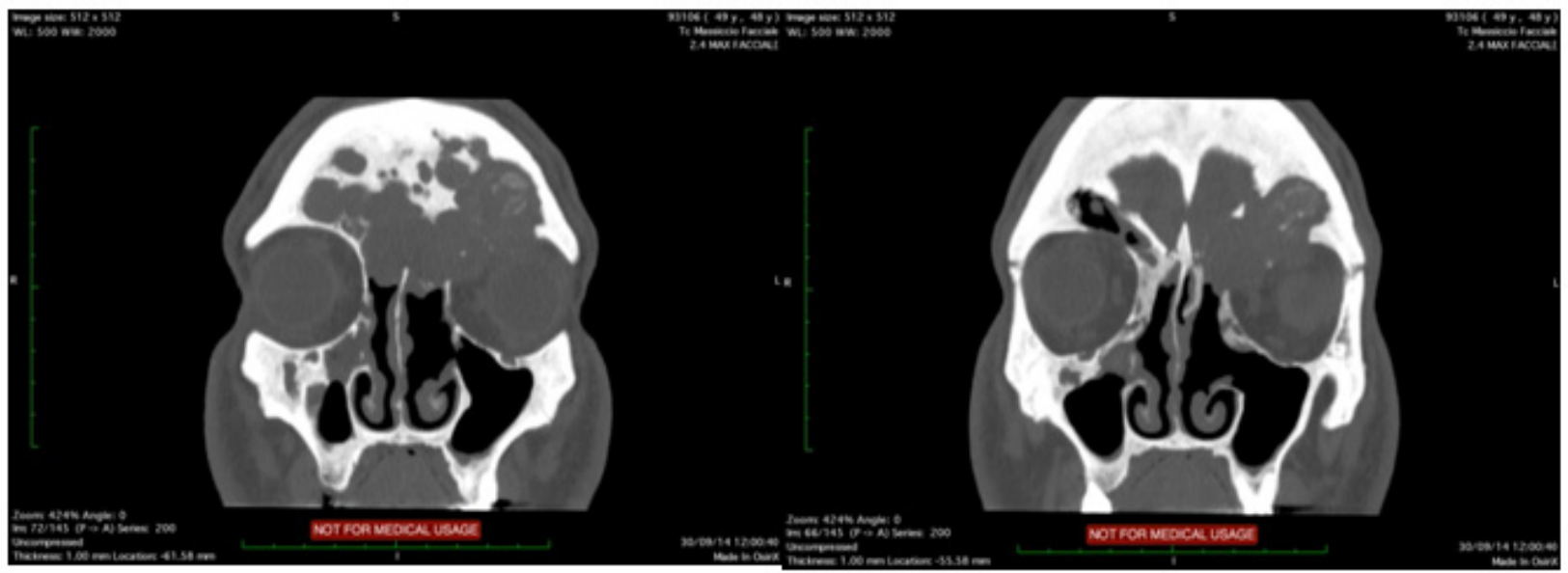

Figure I CT scan coronal view. The lesion reached the left supraorbital region with erosion of the roof of the ipsilateral orbit.

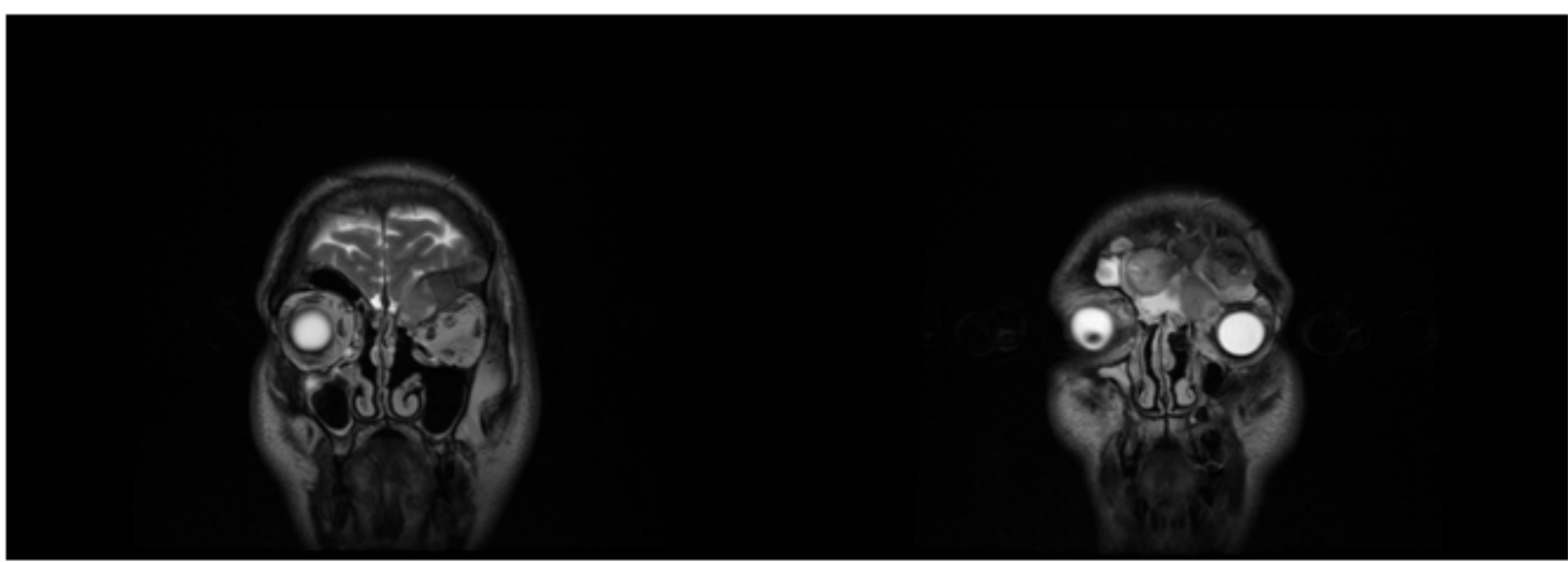

Figure 2 RM scan coronal view. The lesion reached the left supraorbital region with erosion of the roof of the ipsilateral orbit.

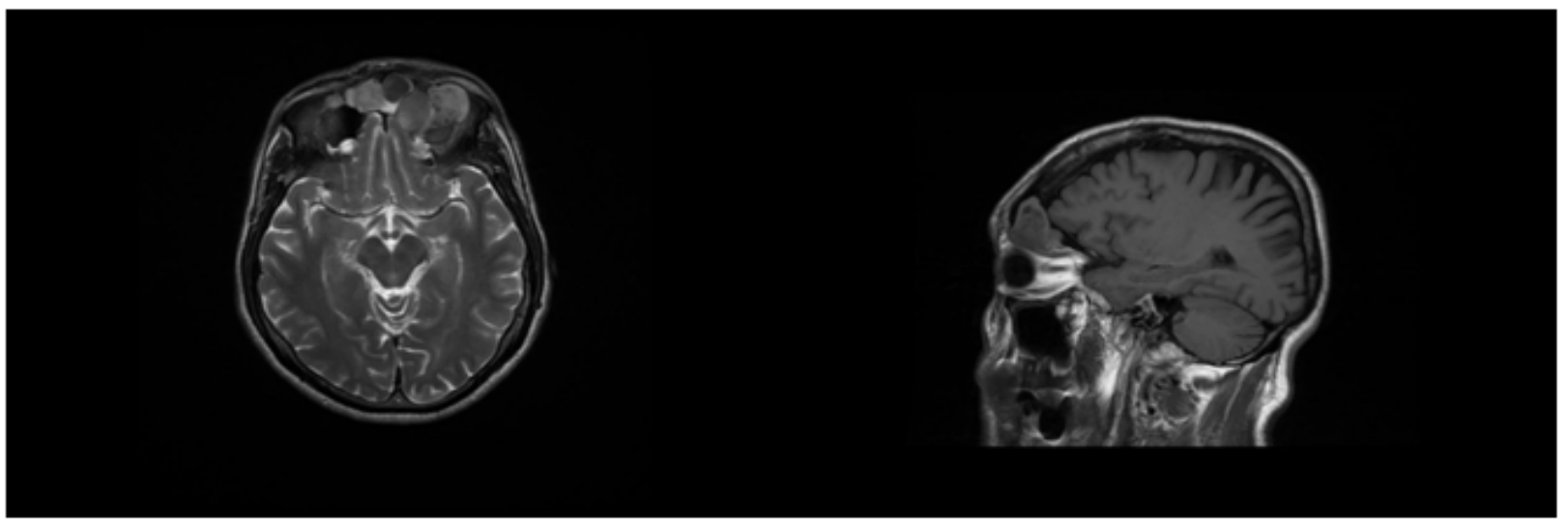

Figure 3 RM scan axial and sagittal view. The dura mater was not involved by mucocele.

Citation: Maria DD, Notaris MD, Malafronte L, et al. Combined endoscopic endonasal and transcranial approach to a recurrent frontal sinus mucocele.J Otolaryngol ENT Res. 2015;2(I):36-39. DOI: 10.15406/joentr.2015.02.00009 


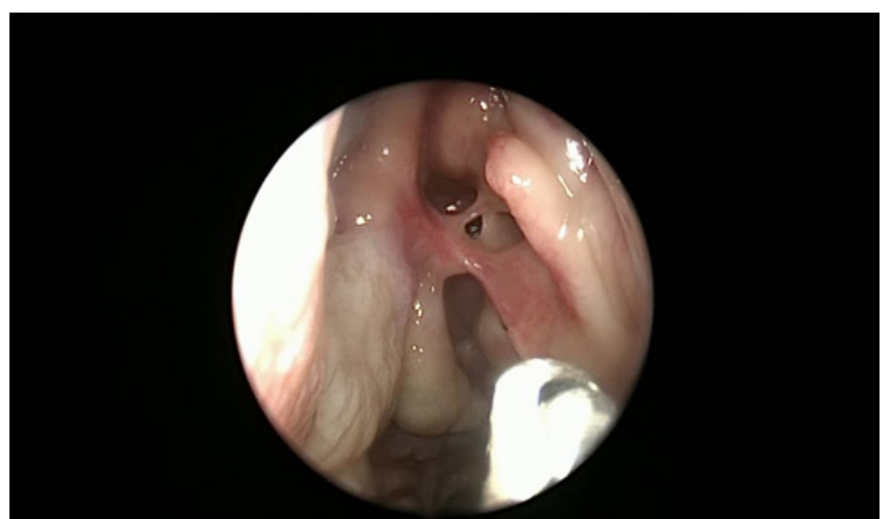

Figure 4 Left synechia at level of nasal-frontal duct.

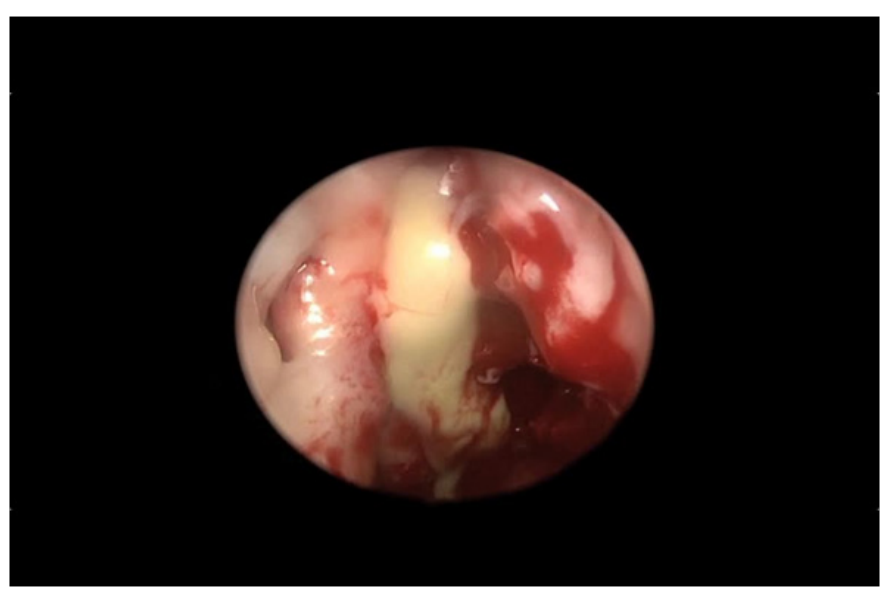

Figure 5 Nasally drainage of mucus-pus.

\section{The essential steps were as follows}

- Bi-coronal incision.

- Setting up of a flap of periosteumuntil the supraorbitalmargin. The preservation of the

- Supraorbital and supratrochlear nerves are required (Figure 6).

- Craniotomydrill and opening from the top of the frontal sinus.

- Removal of the entire pathological mucosa, also by endoscopic control.

- Obliteration of the cavity with abdominalfat (Figure 7).

- Positioning of the periosteal flap filling the frontal sinus above the fat.

- Closing by repositioning the bone flap, scalpsuturing (Figure 8).

During surgery, it was administered to the patient perioperative broad spectrum antibiotic therapy and on awakening he did not present complications so that was conducted in the ENT Division without precautionary transfer to neurointensive unit.

During the six days of postoperative hospitalization the patient had no fever and neurological complications.

The TC postoperative control, performed after 24hours from surgery revealed no intracranial complications but the correct fat positioning in the frontal sinus.

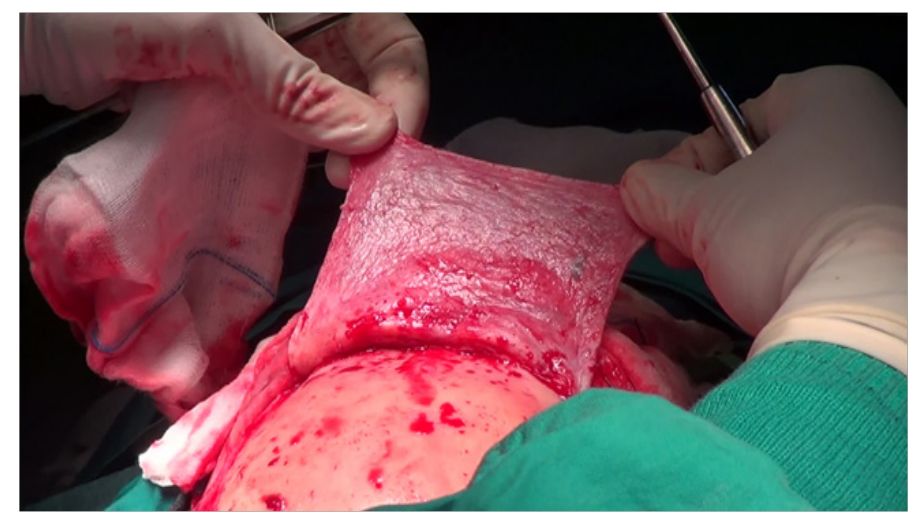

Figure 6 Development of the periosteal (galea) flap.

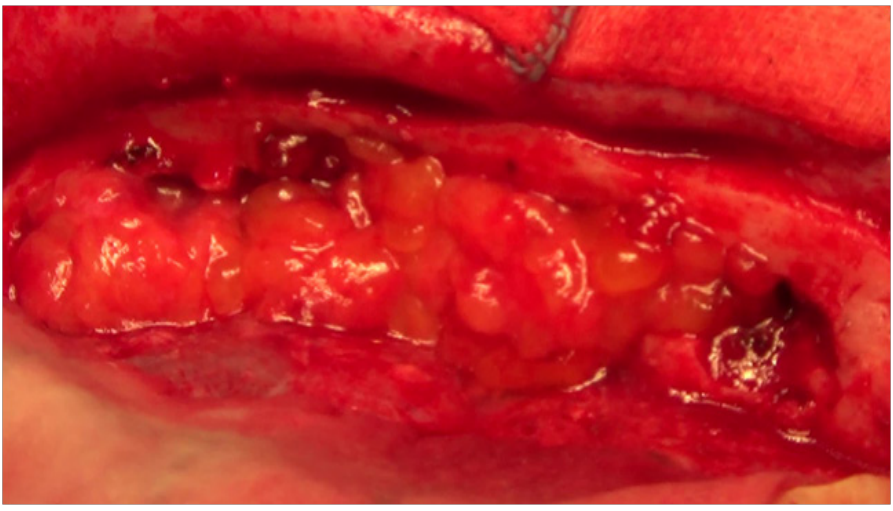

Figure 7 Filling of the sinus cavity with freshly harvested abdominal fat.

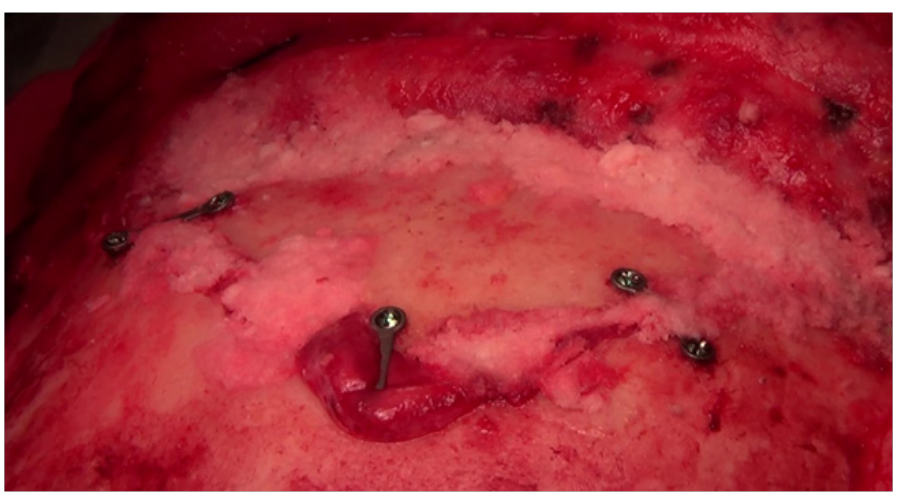

Figure 8 Replacement of the bony flap.

The patient was discharged on the sixth day in excellent conditions. The subsequent endoscopic endonasal control showed no signs of recurrence.

\section{Discussion}

The sinus mucocele is a disease that must be treated only by surgical therapy. The mucocele of the maxillary, ethmoid and sphenoid sinusis easily accessible by endoscopic endonasal approach. When the frontal sinus mucocele is lateral, the endonasal access is in effective to ensure the drainage function, so an extra nasal, transcranial procedure is indicated.

The fundamental objectives of SFO were the following:

1. Management of intra orbital complications by endoscopic 
endonasal approach (FESS).

2. Meticulous removal of the mucocele of the frontal sinus.

3. Closure of the frontal infundibulum. ${ }^{1}$

$\mathrm{CT}$ is essential to assess the extent and localizzation of frontal mucocele, the integrity of the walls of the frontal sinus and the involvement of the other sinuses. It is the map that the surgeon uses during surgery. ${ }^{1}$

The endoscopic approach, in our experience, it is necessary in the forms of mucocele complicated by orbital medial and superior extension.

In fact, the endonasal approach allows easily reaching the mucopurulent cavity in medial and supraorbital area and getting the natural drainage through the nose. Moreover, the entire pathological mucosa present in the intranasal side can be removed and thus avoided the risk of recurrence for residues in this region.

Instead, in the presence of a mega-frontal-sinus, of a lateral extension of the mucocele as well and the clinical history of relapses of the disease is also indicated the transcranial surgical approach. It is therefore necessary an external approach to ensure the meticulous removal of the pathological mucosa. The closure of the naso-frontal duct prevents infection from nasal cavity and displacement of fat into the nose. ${ }^{1}$

So the most suitable material for the obliteration remains abdominal fat, both for its compatibility that even for the purposes of a correct radiological follow-up.

The SFO is a effective method of treatment of frontal sinus mucocele when the entire frontal sinus is not accessible by endonasal endoscopic approach.

Weber et al., ${ }^{6}$ treated 75 primary cases, 7 revisions were necessary for a success rate of $90 \%$.

The SFO can be considered a definitive treatment, but this procedure also has a reported long-term failure rate of up to $18 \%{ }^{2}$

Difficulties interpreting post-operative imaging can also complicate management of patients with persistent symptoms after frontal sinus obliteration. During the postoperativeperiod the MRI isindicated for closeradiological follow-up to monitor the correctpositioning of the fat oearlymucocelerelapses. ${ }^{6}$

The OFSO minimizes the risk of complications with no need for a postoperative neurointensive unit stay compared to the intervention of cranialization. In fact the patient object of this work had a regular postoperative course and was discharged on the sixth day with excellent aesthetic results.

\section{Acknowledgments}

None.

\section{Conflicts of interest}

The authors declare that there are no conflicts of interest.

\section{References}

1. Constantinidis J, Steinhart H, Schwerdtfeger K, et a. Therapy of invasive mucoceles of the frontal sinus. Rhinology. 2001;39(1):33-38.

2. Wormald PJ. Salvage frontal sinus surgery: the endoscopic modified Lothrop procedure. Laryngoscope. 2003;113(2):276-283.

3. Weber R, Draf W, Kratzsch B, et al. Modern concepts of frontal sinus surgery. Laryngoscope. 2001;111(1):137-146.

4. Weber R, Draf W, Keerl R, et al. Current aspects of frontal sinus surgery. II: External frontal sinus operation--osteoplastic approach. HNO. 1995;43(6):358-363.

5. Weber R, Draf W, Keerl R, et al. Aspects of frontal sinus surgery, III: indications and results of osteoplastic frontal sinus operation. HNO. 1995;43(7):414-420.

6. Weber R, Draf W, Keerl R, et al. Osteoplastic frontal sinus surgery with fat obliteration: technique and long-term results using magnetic resonance imaging in 82 operations. Laryngoscope. 2000;110(6):1037-1044.

7. Silverman JB, Gray ST, Busaba NY. Role of osteoplastic frontal sinus obliteration in the era of endoscopic sinus surgery. Int $J$ Otolaryngol. $2012 ; 501896$. 